\title{
A "blanking effect" for surface features: Transsaccadic spatial-frequency discrimination is improved by postsaccadic blanking
}

\author{
Katharina Weiß $^{1,2}$ - Werner X. Schneider ${ }^{1,2}$ - Arvid Herwig ${ }^{1,2}$
}

Published online: 20 May 2015

(C) The Psychonomic Society, Inc. 2015

\begin{abstract}
Although saccadic eye movements occur frequently - about three or four times a second - humans are astonishingly blind to transsaccadic changes. Locational displacements of the saccade target of up to 2 deg of visual angle, and even large changes of a visual scene, can go unnoticed. For a long time, this insensitivity was ascribed to deficits in transsaccadic memory: Only a coarse, (spatially) imprecise representation would be retained across a saccade. This assumption was contradicted by Deubel's and Schneider's (Behavioral and Brain Sciences 17:259-260, 1994) striking finding that locational discrimination performance across a saccade is greatly improved by inserting a short postsaccadic blank. Surprisingly, the question of whether blanking effects occur also for other forms of transsaccadic changes (i.e., surface-feature changes) has been widely ignored. We tested this question by means of a transsaccadic change in spatial frequency. Postsaccadic blanking facilitated spatial-frequency discrimination, but to a smaller amount than the usual blanking effects obtained with locational displacements. This finding bears important implications for models of visual stability and transsaccadic memory.
\end{abstract}

Keywords Blanking effect · Transsaccadic memory · Visual stability

Katharina Weiß

katharina.weiss@uni-bielefeld.de

1 Department of Psychology, Bielefeld University, Bielefeld, Germany

2 Cluster of Excellence "Cognitive Interaction Technology”, Bielefeld University, Bielefeld, Germany
Although saccadic eye movements are ubiquitous - saccades are usually executed three or four times per second-humans are astonishingly insensitive to transsaccadic changes (Bridgeman, Hendry, \& Stark, 1975; Grimes, 1996; Henderson \& Hollingworth, 2003). In particular, they do not notice locational displacements of a stimulus across the saccade of up to $2 \mathrm{deg}$ of visual angle. Amazingly, they can sometimes not even report large changes of the global scene (Bridgeman et al., 1975; Henderson \& Hollingworth, 2003). For many years, these startling findings were interpreted as evidence for a general deficit in transsaccadic memory. It was assumed that only a coarse representation of the presaccadic stimulus is retained across a saccade, lacking detailed and precise stimulus information, including its exact spatial location (e.g., Henderson, 1997, 2008). In the probably most extreme version of this notion, it has even been suggested (O'Regan, 1992; O'Regan \& Noë, 2001) that no information at all has to be retained as an internal representation across saccades. Instead, the world itself would be used as an outside memory that is probed constantly by eye movements.

However, the above-described interpretation of insensitivity to transsaccadic changes has been challenged by a surprising finding: Deubel and colleagues demonstrated that, although under normal conditions participants were hopeless in discriminating displacements across a saccade, they were well able to perform this task if a short postsaccadic blank (50-300 ms) was inserted (cf. Deubel \& Schneider, 1994; Deubel, Schneider, \& Bridgeman, 1996). Interestingly, substantial improvements in locational discrimination accuracy could also be revealed with a postsaccadic shape change (Demeyer, De Graef, Wagemans, \& Verfaillie, 2010), a surface-feature change (Tas, Moore, \& Hollingworth, 2012), or a task-irrelevant displacement of the target orthogonal to the saccade (Wexler \& Collins, 2014). Thus, the existence of the blanking effect demonstrates that poor transsaccadic 
locational discrimination performance is clearly not the result of an imprecise memory representation across the saccade.

But how can this striking improvement in discrimination performance due to a postsaccadic blank be explained? Since the blanking effect relieves insensitivity to transsaccadic changes dramatically, it might be considered the flipside of a mechanism contributing to the impression of transsaccadic visual stability. The retinal position as well as the spatial resolution of an object in the visual field can change dramatically due to saccades. Therefore, the question of how the impression of visual stability is achieved provides a fascinating puzzle for perception research (e.g., Wurtz, Joiner, \& Berman, 2011). Two different types of accounts try to explain how visual stability arises despite frequent eye movements. On the one hand, "cancellation accounts" (e.g., Higgins \& Rayner, 2015) suppose an active process to obtain visual stability. More specifically, they assume that an extraretinal eye movement signal (efference copy/corollary discharge) is used to "cancel out" saccade-induced retinal displacements. On a neural level, predictive remapping of presaccadic locations to the predicted postsaccadic location could actively compensate for retinal displacements caused by the saccade - through either a shift of receptive fields (e.g., Duhamel, Colby, \& Goldberg, 1992) or an activation transfer for attended targets (Cavanagh, Hunt, Afraz, \& Rolfs, 2010; Rolfs, Jonikaitis, Deubel, \& Cavanagh, 2011). A recently discovered alternative to predictive remapping equally could compensate for saccade-induced retinal displacements: presaccadic convergence of receptive fields toward the saccade target (Zirnsak, Steinmetz, Noudoost, Xu, \& Moore, 2014). On the other hand, "assumption" accounts (Wexler \& Collins, 2014) assume that phenomenal visual stability is achieved passively by means of a strong prior assumption of a stable world (e.g., Deubel et al., 1996; Deubel, Schneider, \& Bridgeman, 2002). According to this perspective, the usual human insensitivity to transsaccadic changes occurs due to a strong, adaptive, builtin assumption that the world remains stable during the short time that we need to make a saccade. Unless this assumption is severely violated, transsaccadic object continuity is assumed (Deubel et al., 1996) — at least for the saccade target. Therefore, presaccadic stimulus information will be updated and overwritten with postsaccadic stimulus information (e.g., Schneider, 2013; Tas et al., 2012). But if the assumption of a stable world is broken by a disruption of spatial or temporal object continuity (i.e., a postsaccadic blank, shape change, or surface-feature change), the postsaccadic stimulus is treated as a new object (Kahneman, Treisman, \& Gibbs, 1992; Schneider, 2013) and the presaccadic stimulus information is preserved. Consequently, presaccadic and postsaccadic stimulus information can be compared without difficulty (Schneider, 2013; Tas et al., 2012), and previously neglected extraretinal information will be used (Deubel et al. 1998). Recently, Wexler and Collins (2014) revealed that a task- irrelevant target displacement orthogonal to the saccade leads to effects on displacement discrimination similar to those of a postsaccadic blank, but has no additive effects. They proposed that visual stability is due to an interaction of cancellation and assumption processes: Within the elliptic-shaped region of saccadic errors, visual stability is assumed because it would be adaptive to attribute any displacement within this region to eye movements. Outside this error region visual stability is not assumed, and the efference copy is used to remap pre- and postsaccadic locations. To summarize, cancellation and assumption accounts of visual stability point out that the blanking effect is a valuable tool for investigating mechanisms of visual stability and transsaccadic memory.

Surprisingly, however, the blanking effect has been demonstrated almost exclusively in the locational domain for displacements of the saccade target. To our knowledge, the question of whether a postsaccadic blank supports the discrimination of other transsaccadic changes as well (i.e., a color, shape, or spatialfrequency change) has been addressed only twice. Schneider and Deubel (1996, published conference abstract) found that a postsaccadic blank improved discrimination performance for orientation, shape, and size changes, but not for surface-feature changes as luminance and color. Furthermore, Deubel et al. (2002, Exp.3) reported an improvement of "form change" detection in a black-and-white grid pattern. However, looking more closely, they demonstrated a postsaccadic blanking effect for a location shift in a complex configuration: A square of the Philips pattern changed its position transsaccadically, and the task was to indicate the spatial location of this change (upper or lower part of the pattern). Thus, only the unpublished study by Schneider and Deubel has revealed evidence for blanking effects of visual and nonlocational features such as orientation, shape, and size, but it failed to reveal blanking effects for the surface features color and luminance.

In the present study, we tested whether we could obtain such a nonlocational blanking effect for another kind of surface-feature change, a change in spatial frequency. We decided to investigate a potential blanking effect for surfacefeature discrimination - and more specifically, for spatialfrequency discrimination-for two reasons. First, a potential blanking effect for a surface feature (e.g., spatial frequency) provides a challenge for cancellation accounts of visual stability, because the occulomotor efference copy would probably not contain information about surface-feature changes. Since predictive remapping is the likely candidate for a neural mechanism of cancellation, explaining a (surface) feature-blanking effect in accordance with cancellation accounts presupposes predictive remapping of visual features. However, predictive remapping of visual features other than location and their possible relationship is hotly debated (e.g., Cavanagh et al., 2010; Herwig \& Schneider, 2014; Mathôt \& Theeuwes, 2013; Melcher, 2005, 2007; Rolfs et al., 2011). Evidence for feature remapping has come mostly from reports of adaptation 
aftereffects across saccades. For instance, after short adaptation to a tilted grating at fixation, a test stimulus that is presented at the same location is perceived as being tilted in the opposite direction. If a saccade is made after adaptation, a tiltadaptation aftereffect cannot only be found at retinotopic locations but, critically, also at spatiotopic locations (Melcher, 2005, 2007), indicating remapping of visual features. However, other studies (Afraz \& Cavanagh, 2009; Knapen, Rolfs, Wexler, \& Cavanagh, 2010; Mathôt \& Theeuwes, 2013) have failed to reveal spatiotopic aftereffects, but found only aftereffects of a retinotopic nature. Thus, until visual feature remapping can be demonstrated beyond dispute, a surface-feature blanking effect provides a challenge for cancellation accounts of visual stability. Second, we were especially interested in a spatial-frequency blanking effect, because we have demonstrated recently that the disruption of object continuity by postsaccadic blanking (or a shape change) did not impair the learning of transsaccadic associations between peripheral and foveal spatial-frequency information (Weiß, Schneider, \& Herwig, 2014). Therefore, it is interesting to investigate whether, nevertheless, a postsaccadic blank causes improved transsaccadic spatial-frequency discrimination. This would imply that the mechanisms underlying transsaccadic learning and transsaccadic discrimination are - at least partially—not the same.

\section{Method}

\section{Participants}

Fourteen participants (nine female, five male) with normal or corrected-to-normal vision took part in the experiment. Due to late saccades (i.e., 1,000 ms after target presentation, no saccade was detected), three participants lost either one or two experimental-condition trials, so the statistical analyses were adjusted accordingly.

\section{Apparatus and stimuli}

Participants performed the experiment in a dimly lit room. The viewing distance to a 19-in. display CRT monitor (ViewSonic Graphics Series G90fB) running at $100 \mathrm{~Hz}$ was $71 \mathrm{~cm}$. The resolution of the screen was set to $1,024 \times 768$ pixels, corresponding to the screen's physical dimensions of $36 \mathrm{~cm}$ (width) $\times 27 \mathrm{~cm}$ (height). Eye movements were recorded with a videobased tower-mounted eyetracker (EyeLink1000, SR Research, Ontario, Canada). Eye movements were recorded with a sampling rate of $1000 \mathrm{~Hz}$. The right eye of all participants was monitored, and their heads and chins were stabilized by a head-and-chinrest. During the experiment, a black fixation cross was displayed in the middle of the screen $\left(0.3^{\circ} \times\right.$ $0.3^{\circ}$, line width of two pixels). The stimuli were circular objects $\left(1.5^{\circ}\right)$ filled with sinusoidal gratings of different spatial frequencies $\left(2.45,3.2,3.95,4.7\right.$, and $5.45 \mathrm{cpd}$, orientation $\left.0^{\circ}\right)$. The stimuli were presented on a gray background with a mean luminance of $30 \mathrm{~cd} / \mathrm{m}^{2}$. Examples of the stimuli utilized are shown in Fig. 1.

\section{Procedure}

Before the experiment, a nine-point grid calibration procedure was conducted. After a variable fixation interval (500 to 1, $000 \mathrm{~ms}$ ), each trial started with the presentation of a circular object filled with a vertical sinusoidal grating (orientation $0^{\circ}$ ) to either the left or the right of fixation, $6^{\circ}$ in the periphery. Participants had to saccade to this peripheral stimulus. Critically, on four fifths of the trials, the spatial frequency of this stimulus was changed during the saccade (no-blank trial) or after a short postsaccadic blank of $250 \mathrm{~ms}$ (blank trial). The presaccadic stimulus was defined as the standard stimulus and always had a spatial frequency of 3.95 cycles per degree (cpd). The frequency change was either to a lower $(2.45$, or $3.2 \mathrm{cpd})$ or a higher ( 4.7 or $5.45 \mathrm{cpd}$ ) spatial frequency. On the remaining fifth of the trials, the spatial frequency was not changed $(3.95 \mathrm{cpd})$. The task of the participants was to compare, in a

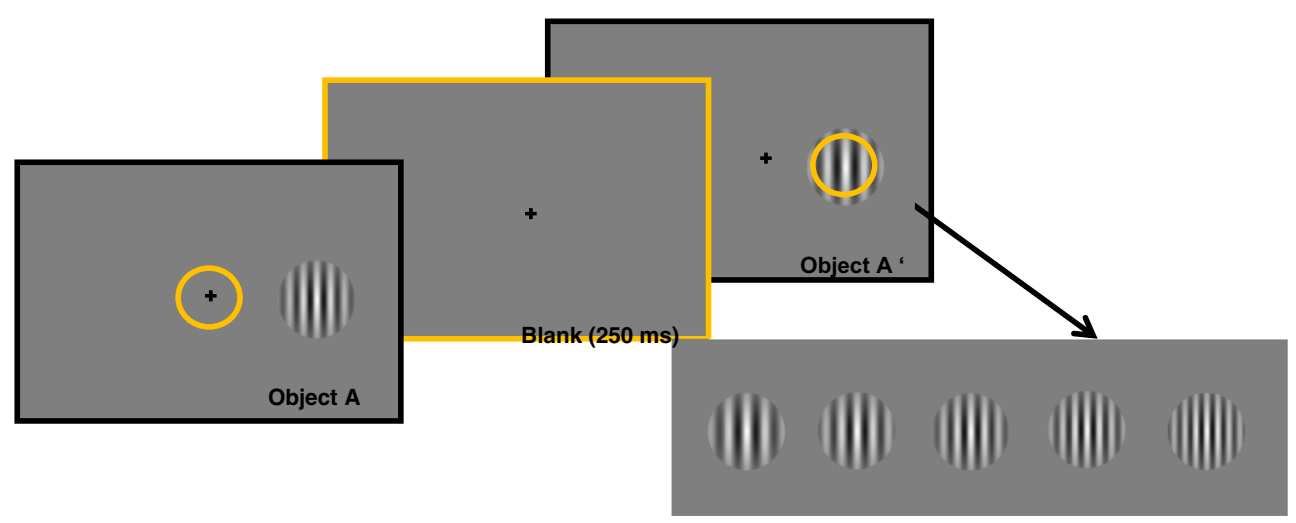

Fig. 1 Example of an experimental trial with a 250-ms blank 
two-alternative forced choice task, the spatial frequencies of the postsaccadic, foveally presented stimulus with the presaccadic peripheral stimulus. ("Are the stripes in the current pattern of stripes wider or narrower than in the previous pattern?"; see Fig. 1). On half of the trials, a 250-ms postsaccadic blank was inserted before the appearance of the postsaccadic stimulus. On the other half of the trials, no blank was inserted and the spatial frequency of the stimulus was changed during the saccade. Additionally, $25 \%$ catch trials of another spatial frequency were inserted, to prevent participants from noticing that the spatial frequency of the presaccadic peripheral stimulus was always $3.95 \mathrm{cpd}$; these catch trials were not analyzed. A session's duration was approximately $45 \mathrm{~min}$. After the saccade, the postsaccadic foveal object was presented for $250 \mathrm{~ms}$ - either immediately (noblank trial) or after a $250-\mathrm{ms}$ postsaccadic blank (blank trial); afterward, it was replaced by a blank of 1,500-ms duration. The experiment consisted of 480 trials (of which 120 were catch trials), which resulted in 36 trials per experimental condition, 2 (blank vs. no blank) $\times 5$ (spatial frequencies). After the experiment, participants were asked in a debriefing whether they had noticed something unusual, whether they had used a conscious strategy to do the task, and how certain they were about their judgments.

\section{Results}

The data were fitted with the Palamedes Toolbox (Prins \& Kingdom, 2009) with a cumulative Gaussian function. Two parameters were obtained: the point of subjective equality (PSE), which denotes the spatial frequency at which both possible frequency judgments ("narrower"/higher vs. "wider"/ lower) are given equally often. In an unbiased judgment, this would be the case if the pre- and postsaccadic stimuli had the same spatial frequency $(3.95 \mathrm{cpd})$. To measure discrimination performance, the difference limen (DL) was computed as half of the frequency change between the .25 and the .75 thresholds. Figure 2 shows the pooled data for blank and no-blank trials for "higher" frequency judgments, and Fig. 3 shows the individual data for each participant in the no-blank condition and the blank condition. A paired $t$ test revealed a significant improvement in discrimination accuracy (DL no blank $M=-0.44$ vs. DL blank $M=-0.21), t(11)=-4.06, p<.01, d=1.17$. The change in PSEs was not significant (PSE no blank $M=4.05$ vs. PSE blank $M=$ 4.05), $t(11)=0.01, p=.99, d=0.02{ }^{1}$

\footnotetext{
${ }^{1}$ Note that the exclusion of Participants 3 and 11, due to a nonconvergence of their fits in the blank condition, would not change the results of the present study: A paired $t$ test reveals better discrimination accuracy for the blank condition $(M=-.23)$ than for the no-blank condition $(M=-.41), t(9)=-$ $3.46, p<.01$.
}

\section{Discussion}

The present study provides evidence for a surface-feature blanking effect: We replicated the classic blanking effect for transsaccadic displacements (cf. Deubel \& Schneider, 1994; Deubel et al., 1996) outside the locational domain with a transsaccadic surface-feature change: a change in spatial frequency. A postsaccadic blank of $250 \mathrm{~ms}$ improved spatialfrequency discrimination in comparison to a no-blank condition. Interestingly, the blanking effect for spatial-frequency discrimination is rather small in direct comparison to the classic blanking effect for locational displacements. However, it is important to note that the blanking effect for spatial-frequency discrimination in the present study provides a conservative estimate of the effect, because the frequency discrimination task was clearly easier than the locational displacement discrimination task usually used. Visual comparison of the psychometric functions in the no-blank control conditions between the present study and Deubel et al. (1996) shows steeper and less biased functions in the present study. Therefore, the postsaccadic blank did not have the same chance to improve frequency discrimination as to improve displacement discrimination. Consequently, the size of the spatial-frequency blanking effect might be underestimated in comparison to the classical blanking effect. ${ }^{2}$

A blanking effect for surface-feature discriminations has several interesting theoretical implications. First of all, it provides a challenge for cancellation accounts, but not for assumption accounts, of visual stability. Whether cancellation accounts can meet this challenge depends critically on the existence of predictive remapping of visual features or remapping of spatial locations that also allows for visual feature updating at these locations - for example, by pointers toward the respective feature maps (e.g., Melcher \& Colby, 2008). However, as we stated already in the introduction, the idea of visual feature remapping and its possible relationship with remapping of spatial locations is highly controversial, and the debate is far from settled, yet (e.g., Cavanagh et al., 2010; Melcher, 2007; Rolfs et al., 2011; for a discussion of how location and visual feature prediction across saccades could be related, see Herwig \& Schneider, 2014).

A surface-feature blanking effect indirectly supports assumption accounts because it is in line with the theory of "task-driven visual attention and working memory" (TRAM; Schneider, 2013). TRAM addresses the role of visual attention across different fixations in a biased-competition approach. Importantly, it assumes that the testing of object continuity/correspondence

\footnotetext{
${ }^{2}$ Note that in terms of Cohen's $d$, our study revealed a large effect (effect size $>0.8$ ), but visual inspection of the psychometric functions in Deubel et al.'s (1996) study denotes that the original blanking effect is also a "large" effect, with probably a considerable higher numerical value.
} 


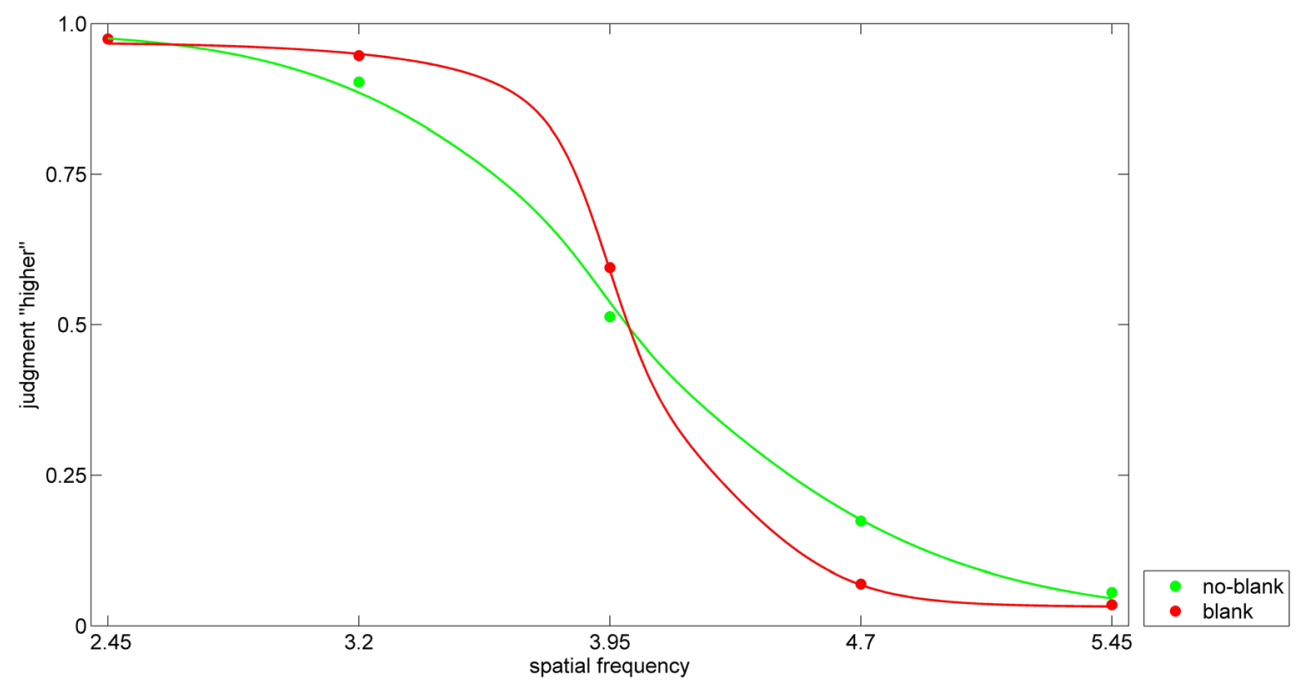

Fig. 2 Mean "higher" spatial-frequency judgments, as a function of the postsaccadic target's spatial frequency

plays a crucial role for computation of attentional priorities across a saccade — or to put it otherwise, testing of the assumption of a stable world. Object correspondence is confirmed if the visual input of the postsaccadic stimulus corresponds to the input predicted from the presaccadic stimulus information. As a result of confirmation, presaccadic information is updated and overwritten by postsaccadic information. Consequently, transsaccadic displacement or spatialfrequency discrimination should be impaired. If, on the other hand, an object correspondence failure is signaled due to a postsaccadic blank or shape change, the presaccadic stimulus information should be retained, encapsulated, and not overwritten, so that transsaccadic discrimination performance should be massively improved. Thus, via an object correspondence testing mechanism, TRAM explains the classical blanking effect for locational displacements and predicts a spatialfrequency blanking effect.

Furthermore, this spatial-frequency blanking effect implies that transsaccadic learning and transsaccadic discrimination rely on
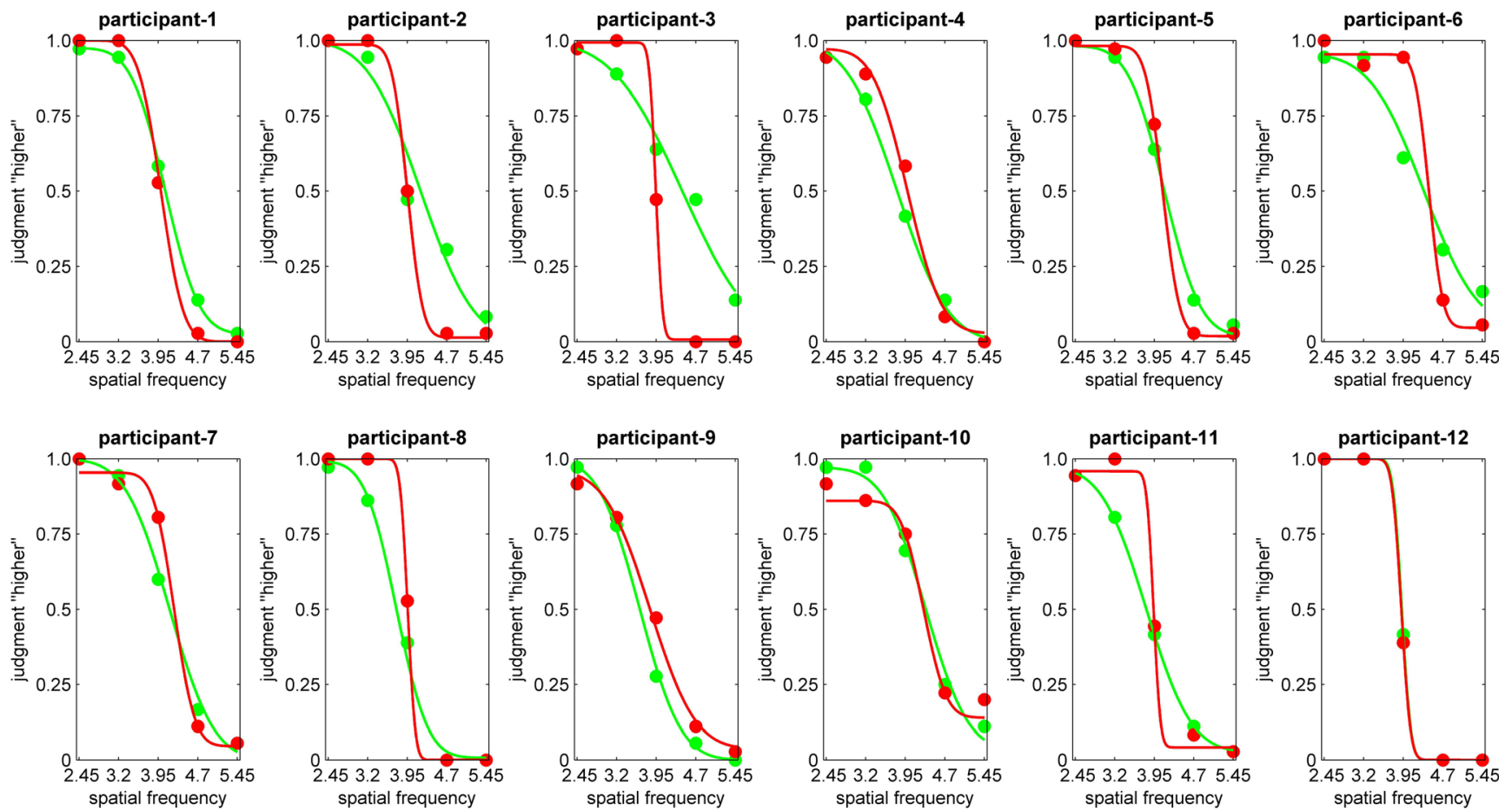

Fig. 3 Frequencies of the "higher" spatial-frequency judgments for each individual participant 
different mechanisms, because transsaccadic learning of spatialfrequency associations is not affected by the disruption of object continuity through blanking, although transsaccadic frequency discrimination is improved by the blank (Weiß et al., 2014). Thus, object continuity testing plays a critical role in transsaccadic discrimination, but not in transsaccadic learning. This supports the idea that transsaccadic learning acts as a default mode of the visual system (Herwig \& Schneider, 2014; Weiß et al., 2014): Previously learned associations between foveal and peripheral object appearances allow the visual system to make predictions about the foveal and peripheral visual input, facilitating object recognition and visual search. These predictions contribute to the perception of visual stability by compensating for the visual system's inhomogeneity. Finally, it is important to note that it is unlikely that the blanking effect for spatial-frequency changes can be explained by an enhancement of the perceived spatial frequency due to attention (Abrams, Barbot, \& Carrasco, 2010; Gobell \& Carrasco, 2005). The blanking manipulation was applied postsaccadically to the foveated target, whereas enhancement effects on spatial-frequency perception have only been found with peripheral stimuli.

To summarize, we report first evidence of a robust blanking effect for spatial-frequency discrimination - a surface-feature change. This finding bears interesting implications for theories of visual stability and transsaccadic memory. It provides a challenge for cancellation accounts of visual stability, but not for assumption accounts. Whereas the challenge for cancellation accounts could only be met by predictive remapping of visual features, a surface-feature blanking effect is predicted by TRAM - a model that incorporates object correspondence testing, that is testing the assumption of a stable world. Furthermore, a spatial-frequency blanking effect indicates that transsaccadic discrimination and transsaccadic learning rely on different mechanisms. Thus, surface-feature blanking effects provide a useful tool to investigate transsaccadic memory and visual stability.

Author note This work was supported by a grant of the Excellence Cluster "Cognitive Interaction Technology (CITEC)" to W.X.S., and by a grant from the German Research Council (Deutsche Forschungsgemeinschaft; DFG) to A.H. and W.X.S. (No. He6388/1-1).

\section{References}

Abrams, J., Barbot, A., \& Carrasco, M. (2010). Voluntary attention increases perceived spatial frequency. Attention, Perception, \& Psychophysics, 72, 1510-1521. doi:10.3758/APP.72.6.1510

Afraz, A., \& Cavanagh, P. (2009). The gender-specific face aftereffect is based in retinotopic not spatiotopic coordinates across several natural image transformations. Journal of Vision, 10(10), 1-17. doi:10. $1167 / 9.10 .10$
Bridgeman, B., Hendry, D., \& Stark, L. (1975). Failure to detect displacement of the visual world during saccadic eye movements. Vision Research, 15, 719-722.

Cavanagh, P., Hunt, A. R., Afraz, A., \& Rolfs, M. (2010). Visual stability based on remapping of attention pointers. Trends in Cognitive Sciences, 14, 147-153. doi:10.1016/j.tics.2010.01.007

Demeyer, M., De Graef, P., Wagemans, J., \& Verfaillie, K. (2010). Object form discontinuity facilitates displacement discrimination across saccades. Journal of Vision, 10(6), 17. doi:10.1167/10.6.17. 1-14.

Deubel, H., Bridgeman, B., \& Schneider, W. X. (1998). Immediate postsaccadic information mediates space constancy. Vision Research, 5, 3147-3159.

Deubel, H., Bridgeman, B., \& Schneider, W. X. (2004). Different effects of eyelid blinks and target blanking on saccadic suppression of displacement. Perception \& Psychophysics, 66, 772-778. doi:10.3758/ BF03194971

Deubel, H., \& Schneider, W. X. (1994). Perceptual stability and postsaccadic visual information: Can man bridge a gap? Behavioral and Brain Sciences, 17, 259-260. doi:10.1017/ S0140525X00034397

Deubel, H., Schneider, W. X., \& Bridgeman. (1996). Postsaccadic target blanking prevents saccadic suppression of image displacement. Vision Research, 36, 985-996.

Deubel, H., Schneider, W. X., \& Bridgeman, B. (2002). Transsaccadic memory of position and form. Progress in Brain Research, 140, 165-180. doi:10.1016/S0079-6123(02)40049-0

Duhamel, J. R., Colby, C. L., \& Goldberg, M. E. (1992). The updating of the representation of visual space in parietal cortex by intended eye movements. Science, 255, 90-92. doi:10.1126/science. 1553535

Gobell, J., \& Carrasco, M. (2005). Attention alters the appearance of spatial frequency and gap size. Psychological Science, 16, 644-651.

Grimes, J. (1996). On the failure to detect changes in scenes across saccades. In K. Akins (Ed.), Perception (Vancouver Studies in Cognitive Science, Vol. 5 (pp. 89-110). Oxford, UK: Oxford University Press.

Henderson, J. M. (1997). Transsaccadic memory and integration during real-world object perception. Psychological Science, 8, 51-55.

Henderson, J. M. (2008). Eye movements and scene memory. In S. J. Luck \& A. Hollingworth (Eds.), Visual memory (pp. 87-121). Oxford, UK: Oxford University Press.

Henderson, J. M., \& Hollingworth, A. (2003). Global transsaccadic change blindness during scene perception. Psychological Science, 14, 493-497. doi:10.1111/1467-9280.02459

Herwig, A., \& Schneider, W. X. (2014). Predicting object features across saccades: Evidence from object recognition and visual search. Journal of Experimental Psychology: General, 143, 1903-1922.

Higgins, E., \& Rayner, K. (2015). Transsaccadic processing: Stability, integration, and the potential role of remapping. Attention, Perception, \& Psychophysics, 77, 3-27. doi:10.3758/s13414-0140751-y

Kahneman, D., Treisman, A., \& Gibbs, B. J. (1992). The reviewing of object files: Object-specific integration of information. Cognitive Psychology, 24, 175-219. doi:10.1016/ 0010-0285(92)90007-O

Knapen, T., Rolfs, M., Wexler, M., \& Cavanagh, P. (2010). The reference frame of the tilt aftereffect. Journal of Vision, 10(1), 8-1-13. doi:10. $1167 / 10.1 .8$

Mathôt, S., \& Theeuwes, J. (2013). A reinvestigation of the reference frame of the tilt-adaptation aftereffect. Scientific Reports, 3(1152), 1-6. doi:10.1038/srep01152

Melcher, D. (2005). Spatiotopic transfer of visual-form adaptation across saccadic eye movements. Current Biology, 15, 1745-1748. doi:10. 1016/j.cub.2005.08.044

Melcher, D. (2007). Predictive remapping of visual features precedes saccadic eye movements. Nature Neuroscience, 10, 903-907. doi: $10.1038 / \mathrm{nn} 1917$ 
Melcher, D., \& Colby, C. L. (2008). Trans-saccadic perception. Trends in Cognitive Sciences, 12, 466-473. doi:10.1016/j.tics.2008.09.003

O’Regan, J. K. (1992). Solving the "real" mysteries of visual perception: The world as an outside memory. Canadian Journal of Psychology, 46, 461-488.

O'Regan, J. K., \& Noë, A. (2001). A sensorimotor account of vision and visual consciousness. Behavioral and Brain Sciences, 24, 939-973. doi:10.1017/S0140525X01000115. disc. 973-1031.

Prins, N., \& Kingdom, F.A.A. (2009). Palamedes: Matlab routines for analyzing psychophysical data [Software]. Retrieved from www. palamedestoolbox.org

Rolfs, M., Jonikaitis, D., Deubel, H., \& Cavanagh, P. (2011). Predictive remapping of attention across eye movements. Nature Neuroscience, 14, 252-256. doi:10.1038/nn.2711

Schneider, W. X. (2013). Selective visual processing across competition episodes: A theory of task-driven visual attention and working memory. Philosophical Transactions of the Royal Society B, 368, 20130060. doi:10.1098/rstb.2013.0060. 1-13.

Schneider, W. X., \& Deubel, H. (1996). Transsaccadic memory: A postsaccadic gap enhances the perception of intrasaccadic object changes differently for dorsal and ventral representations [Abstract]. Perception, 25(5, ECVP Abstract Supplement)

Tas, A. C., Moore, C. M., \& Hollingworth, A. (2012). An objectmediated updating account of insensitivity to transsaccadic change. Journal of Vision, 12(11), 18. doi:10.1167/12.11.18. $1-13$.

Weiß, K., Schneider, W. X., \& Herwig, A. (2014). Associating peripheral and foveal visual input across saccades: A default mode of the human visual system. Journal of Vision, 14(11), 7. doi:10.1167/14.11. 7. $1-15$.

Wexler, M., \& Collins, T. (2014). Orthogonal steps relieve saccadic suppression. Journal of Vision, 14(2), 13. doi:10.1167/14.2.13. 1-9.

Wurtz, R. H., Joiner, W. M., \& Berman, R. A. (2011). Neuronal mechanisms for visual stability: Progress and problems. Philosophical Transactions of the Royal Society B, 366, 492-503. doi:10.1098/ rstb.2010.0186

Zirnsak, M., Steinmetz, M. A., Noudoost, B., Xu, K. Z., \& Moore, T. (2014). Visual space is compressed in prefrontal cortex before eye movements. Nature, 507, 504-507. 